Mediterránea Ser. Biol. (1990), n. ${ }^{\circ}$ 12. Pág. 59-70

\title{
SOBRE LA PRESENCIA DE PSEUDOSCORPIONES EN LA DIETA DE SAURIOS PSAMÓFILOS DE LA PROVINCIA DE ALICANTE
}

\author{
por \\ JUAN A. ZARAGOZA MIRALLES ${ }^{(1)}$ Y EDUARDO SEVA ROMÁN ${ }^{(1)}$
}

\section{RESUMEN}

Se cita la presencia de dos especies de pseudoscorpiones (Olpium pallipes e Hysterochelifer sp.) en los contenidos estomacales y heces de Acanthodactylus erythrurus y Psammodromus algirus en dunas de la provincia de Alicante. Se confïma la relativa abundancia de la primera de ellas sobre ramas y hojas de Thymelaea hirsuta, mediante mangueos mensuales y se discute la estrategia alimentaria estacional que permite la incorporación de las mismas a la dieta de los saurios. Asimismo, se cita la presencia de «Sinnesborsten» en los dedos de la pinza del pedipalpo de $O$. pallipes y se revisan algunos caracteres del género.

\section{SUMMARY}

The presence of two pseudoscorpions species (Olpium pallipes and Hysterochelifer sp.) in the stomach contents and feaces from Acanthodactylus erythrurus and Psammodromus algirus from sand dunes in Alicante's province (Spain), are cited. The relative abundance of the first one on Thymelaea hirsuta branches and leaves is confirmed by monthly net sweeping, and the seasonal feeding strategy that leads them to get included in the sauria diet is discussed. Likewise, the presence of «Sinnesborsten» in the pedipalp claw fingers of $O$. pallipes is mentioned and some genera characters reviewed.

Palabras clave: Acanthodactylus erythrurus, Psammodromus algirus, Olpium pallipes, Hysterochelifer sp., arenal costero, contenidos estomacales.

Key words: Acanthodactylus erythrurus, Psammodromus algirus, Olpium pallipes, Hysterochelifer sp., coastal dunes, stomach contents.

(1) Departamento de Ciencias Ambientales y Recursos Naturales. Universidad de Alicante. Apdo. 99. 03080 Alicante. 


\section{INTRODUCCIÓN}

\section{Olpium pallipes $(\mathrm{H}$. Lucas)}

Material: contenidos estomacales de Acanthodactylus erythrurus (Schinz).

A.e. (M) n. ${ }^{\circ}$ 3, AS(L), 26.V.78, E. Seva leg.: 1 ejemplar adulto; A.e. (M) n. ${ }^{\circ} 12$ (b), W(L), 02.VI.78, E. Seva leg.: 2 ejs.(?); A.e. (M) n. ${ }^{\circ} 28$ (11 niñas), ?, E. Seva leg.: 3 ejs. (al menos uno adulto); A.e. (M) n. ${ }^{\circ} 13$, W L, 28.VI.78, E. Seva leg: 1 ej. adulto; A.e. (M) n. ${ }^{\circ} 2$, ?, E. Seva leg.: 3 ejs. adultos; A.e. n. ${ }^{\circ} 32$, 05.V.82, E. Seva leg.: 2 ejs. adultos; A.e. n. ${ }^{\circ} 24$, 11.VI.82, E. Seva leg.: 1 ej. adulto; A.e. (sin numeración), 25.IV.82, E. Seva leg.: 1 ej. (?): A.e. n. ${ }^{\circ}$ 9, 10.VIII.82, E. Seva leg.: 12 ejs.: 6 tritoninfas, 6 adultos.

Material: contenidos de heces de Acanthodactylus erythrurus (Schinz):

A.e. n. ${ }^{\circ}$ 31. 20.VI.82, E. Seva leg.: 1 ej. adulto; A.e. n. ${ }^{\circ}$ 9, 10.VIII.82, E. Seva leg.: 2 ejs. adultos; A.e. n. ${ }^{\circ} 68,25 . I V .82$, E. Seva leg.: 1 ej. de $O l$ pium pallipes? (determinado sobre una sola pata delantera de pseudoscorpión).

La determinación de los restos arriba citados como pertenecientes a la especie Olpium pallipes ha sido efectuada por comparación de las estructuras de ejemplares capturados directamente, mediante mangueo en los arbustos. El $n .^{\circ}$ de ejemplares presentes en cada tubo ha sido establecido por las piezas sueltas encontradas (en su mayoría, un solo escudo prosómico, un solo pedipalpo o una única pinza), atendiendo a la simetría bilateral, a su diferente grado de desarrollo ninfal y a su tamaño.

El contenido de los tubos marcados con E. Seva como recolector pertenece a la zona de playa de El Saladar. El resto, presumiblemente también, aunque no puede confirmarse.

Ejemplares capturados por E. Seva mediante mangueo de ramas bajas de Thymelaea hirsuta, presentes como discretamente abundantes en la zona estudiada de El Saladar: -.V.87: 1 hembra; -.V.87: 1 hembra; 21.VIII.87: 1 deutoninfa (D), 1 tritoninfa (T), 1 macho; -.I.88: 3 hembras; 27.IV.87: 4 D, 3 T; 27.VI.87: $1 \mathrm{~T}, 1$ hembra.

Contenidos estomacales de Psammodromus algirus (L.):

P.a. n. ${ }^{\circ}$ 25, 22.IV.82, El Saladar, E. Seva leg.: 1 ej. (?); P.a. n. ${ }^{\circ} 30$, ?, El Saladar, E. Seva leg.: 1 ej. adulto, junto a restos de 2 ejs. de Hysterochelifer sp.

Los ejemplares citados coinciden aceptablemente con la redescripción de la especie, efectuada por HEURTAULT (1979). Los pedipalpos en algunos especímenes son algo más esbeltos, así como la relación longitud/anchura del escudo prosómico.

Estos ejemplares presentan las características típicas del género: escudo prosómico no excesivamente más largo que ancho; abdomen no demasiado alargado y algo engrosado; los terguitos, a excepción de los dos primeros, bien esclerotizados; tricobotrio it netamente distal de est, st alejado de $b$ y $s b$; fémur del pedipalpo con dos sedas pseudotactiles (fig. 3); la quetotaxia del basitarso de la pata IV, provisto de tres pares de sedas laterales internas, 2 sedas ventrales y 2 dorsales; telofémur del primer par de patas, claramente más corto que el basifémur; canales del veneno en la pinza de corta longitud. Pero, además, presentan algunos caracteres no frecuentes o inéditos para el género: el escudo prosómico (figs. 1 y 2) posee un surco basal bien visible en todos los ejemplares (definido en el género) e, igualmente, otro medial que en algu- 

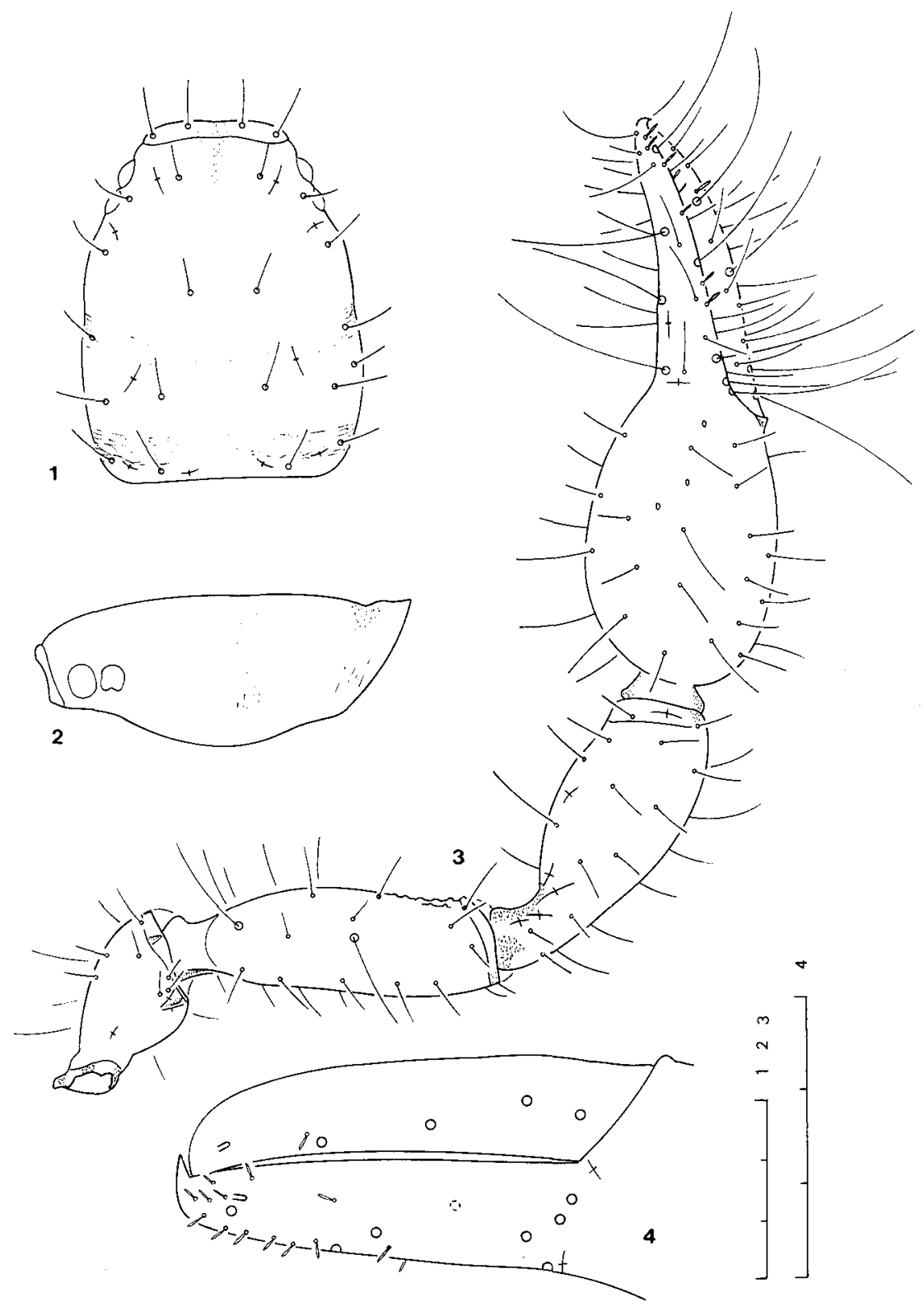

Figuras 1-4.- Olpium pallipes (H. Lucas): 1. Escudo prosómico, visión dorasl; 2. Id., visión lateral; 3. Pedipalpo; 4. Extremo de la pinza del pedipalpo, visión lateral. 
nos individuos es muy evidente y en otros está más difuminado (raramente descrito); el dedo fijo de la pinza del pedipalpo está provisto de diversas sedas cortas no puntiagudas «Sinnesborsten», más abundantes hacia el extremo del dedo (fig. 4).

La confrontación de estos dos últimos caracteres entre los tres géneros de Olpiinae presentes en la Europa occidental: Olpium, Minniza y Calocheiridius, nos lleva a las siguientes conclusiones: la presencia de dos surcos prosómicos no puede, en general, ser utilizada como carácter distintivo entre Minniza o Calocheiridius frente a Olpium; la posesión de sedas sensoriales en el dedo fijo de la pinza, hasta ahora creída como característica de Calocheiridius y otros géneros tropicales, es común a los tres géneros citados (observaciones del autor han comprobado que se hallan, asimismo, presentes en Minniza).

La coincidencia de caracteres propios tanto de Olpium como de Minniza en una misma especie: Olpium minnizoides Vachon, revela la vecindad de ambos géneros y las dificultades para separarlos (VACHON, 1966).

Los trabajos de HEURTAULT (1976, 1980) y de MAHNERT (1982) ponen de manifiesto que, conforme se avanza en el conocimiento de los diversos géneros de la familia Olpiidae, se hace más difícil la definición de los mismos, reduciendo en muchos casos las diferencias, anteriormente a nivel genérico, a simples variaciones específicas.

La especie es nueva para el País Valenciano. Conocida en la Península Ibérica de Portugal y de las provincias españolas de Málaga, Cádiz, Sevilla, Madrid, Ciudad Real y Pontevedra. En las Baleares se la encuentra en Mallorca y Menorca (Fig. 5).

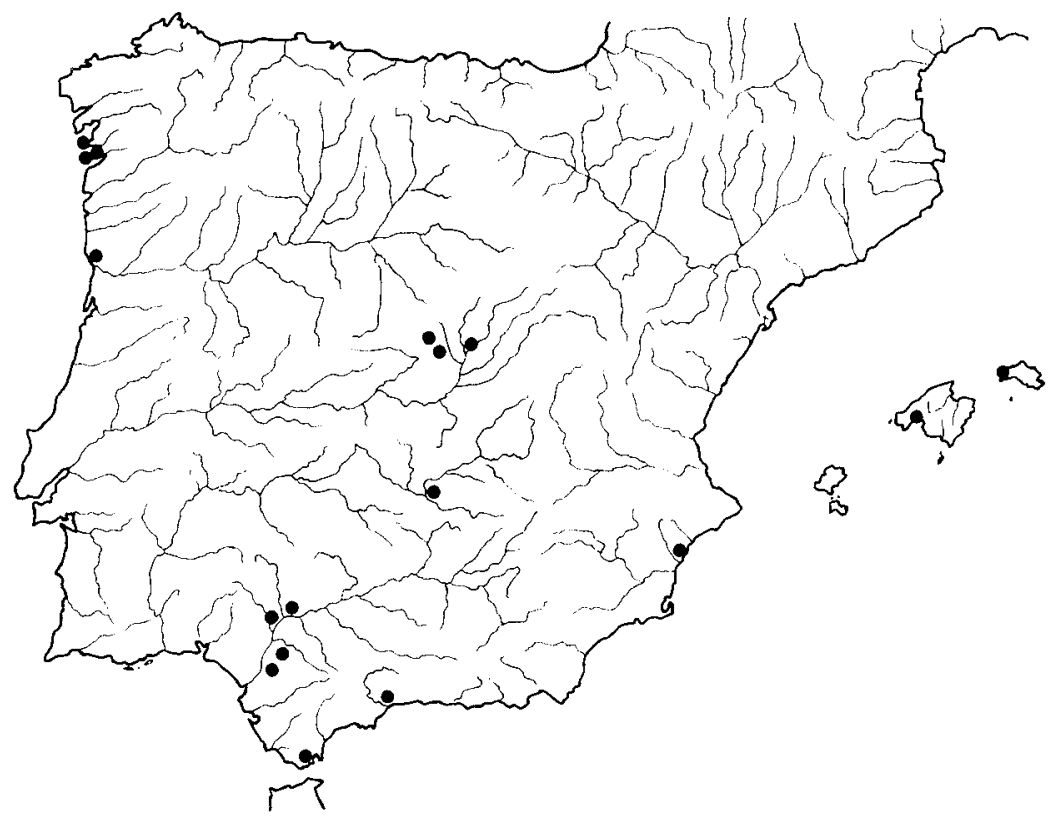

Figura 5.- Distribución conocida de Olpium pallipes (H. Lucas) en Ia Península Ibérica e Islas Baleares. 
Se la localiza en las zonas arenosas cercanas a la línea de costa, bajo piedras o entre la vegetación.

Hysterochelifer sp.

Material: contenido estomacal de Psammodromus algirus (L.).

P.a. n. ${ }^{\circ}$ 30, ?, El Saladar, E. Seva leg.: 2 ejs. adultos, junto a restos de 1 ej. de Olpium pallipes.

Los restos de estos especímenes se limitan a dos pedipalpos casi completos, otras dos pinzas y un fémur de otro individuo, dos pellejos abdominales con los terguitos incompletos y una tibia y un tarso de la pata I de un ejemplar macho.

La fortuna de haber hallado en el contenido del tubo parte de la pata delantera de un macho, ha permitido la determinación del género al que corresponden estos ejemplares. La presencia de una espina subapical en el tarso (Fig. 7) nos obliga a su inclusión dentro de las especies del género Hysterochelifer Chamberlin.

Los escasos restos hallados dan pie, tan sólo, a una breve descripción:

Terguitos con 6/7 sedas sobre cada hemiterguito. Expansiones laterales poco pronunciadas.

Pedipalpos (fig. 6) finalmente granulados, trocánter, tibia y fémur con gruesos tubérculos pilígeros. Fémur $3.92 x$ veces más largo que ancho; tibia $2.95 \mathrm{x}$; mano con mango $2.36 \mathrm{x}$, sin el mango $2.10 \mathrm{x}$; el dedo $1.59 \mathrm{x}$ más corto que la mano con mango y $1.42 \mathrm{x}$ que sin el mango; la pinza con mango $3.68 \mathrm{x}$, sin el mango 3.44x. Dedo fijo con 38/41 dientes hasta distal de esb, el primero pequeño, los $7 / 10$ basales más redondeados. Dedo móvil con 38/42 dientes hasta la altura de $s b$, los dos primeros pequeños, los $7 / 8$ basales redondeados. Nodus ramosus en el dedo fijo basal de et, a mitad de camino entre et/est, en el dedo móvil hasta poco distal de $t$.

Pata I con un telotarso provisto de una prominente espina subapical. Uñas disimétricas, una de ellas bifurcada. Seda subterminal dentada.

Medidas (en milímetros):

Fémur: 0.781/0.199.

Tibia: $0.722 / 0.245$.

Mano con mango: $0.781 / 0.331$.

Mano sin mango: L. 0.695 .

Dedo: 0.490 .

Aun con los pocos datos que suministra lo fragmentario de los restos encontrados, creemos poder atribuir estos ejemplares a la especie Hysterochelifer tuberculatus (H. Lucas) por concurrir los siguientes caracteres: dedo de la pinza claramente más corto que la mano sin mango, seda subterminal del tarso dentada, sedas normales sobre el lado interno de la tibia y el fémur, ist poco proximal de est.

Mayor dificultad tiene poder asignarlos a cualquiera de las dos razas presentes en la Península Ibérica: $H$. tuberculatus tuberculatus (H. Lucas) y $H$. tuberculatus ibericus (Beier), con las que comparte algunas medidas y proporciones. La posición de ist respecto a isb parece aproximarse más a ibericus, ello si aún consideramos la diferenciación de la especie en subespecies geográficas 0 , por el contrario, admitimos que se trata de variaciones de la especie típica (CALLAINI, 1986). 


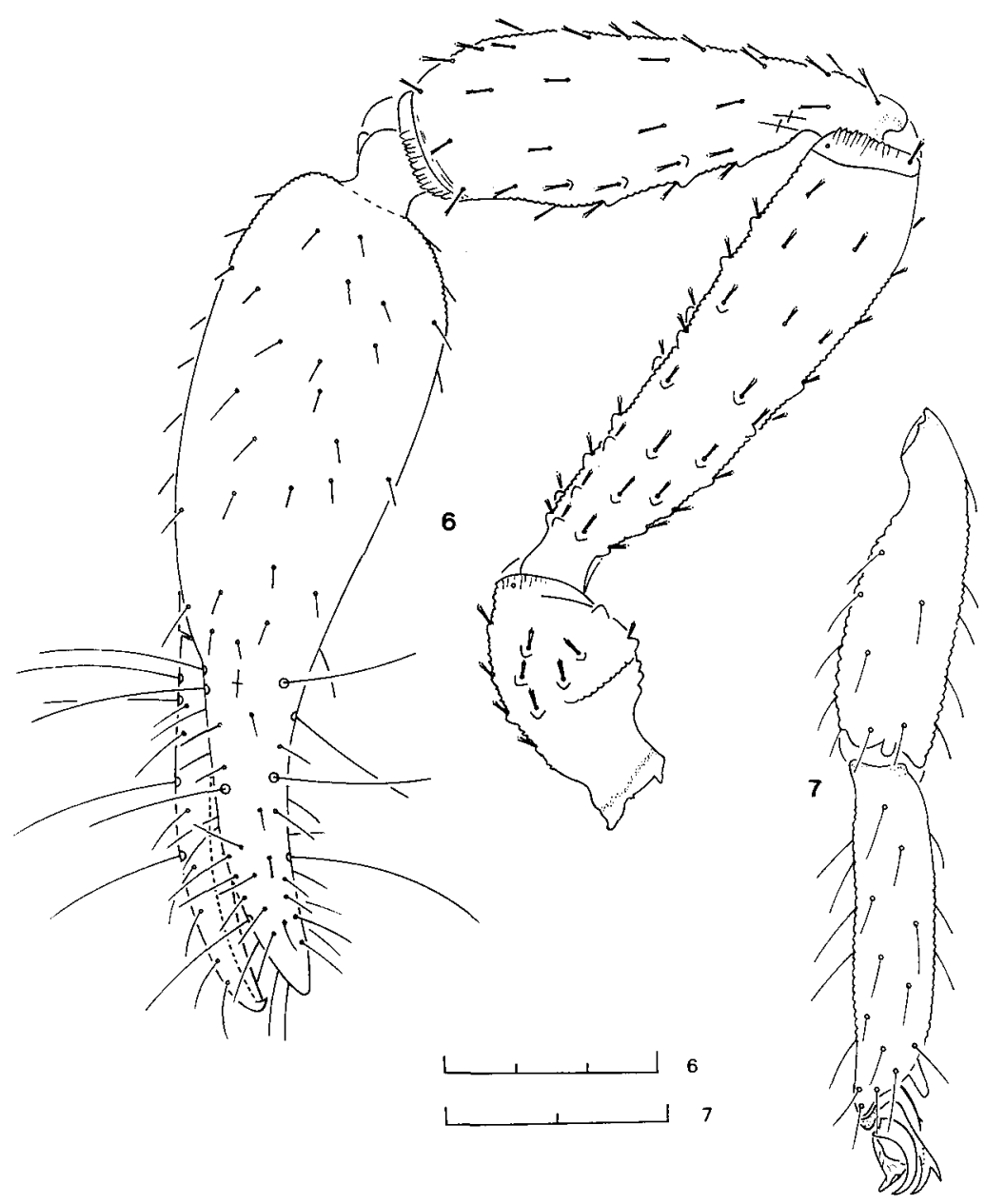

Figuras 6-7.-Hysterochelifer sp.: 6. Pedipalpo; 7. Extremo pata I.

Esta especie mediterránea es frecuente en las provincias españolas del Centro, Sur y Este de la Península Ibérica: Madrid, Ciudad Real, Guadalajara, Cádiz, Sevilla, Granada, Málaga, Córdoba, Gerona, Tarragona, Valencia e, igualmente, en las Baleares. La cita es nueva para la provincia de Alicante.

\section{Predación por vertebrados}

Podemos calificar de escasos los estudios sobre determinados aspectos ecológicos en los pseudoscorpiones. Especialmente poco se ha profundizado sobre la naturaleza de sus presas y enemigos. 
Acerca de la alimentación de los pseudoscorpiones, salvo algún trabajo general como el de GILBERT (1951), lo que existe es un buen número de breves apuntes, la mayoría sobre situaciones individuales y muchas de ellas llevadas a cabo en el laboratorio en condiciones precarias y, posiblemente, con presas no habituales. En general se puede afirmar que los pseudoscorpiones se alimentan preferentemente de insectos u otros artrópodos de pequeño tamaño y/o de sus larvas, dándose algunos casos de canibalismo.

Mucho mejor conocido en el comportamiento de este grupo, y sobre ello existe una extensa bibliografía, es el fenómeno de foresia o el transporte de un lugar a otro utilizando otro animal al que se adhieren uno o varios individuos; generalmente se trata de insectos voladores como dípteros, coleópteros o himenópteros, aunque también utilizan vertebrados como aves e, incluso, mamíferos como los topos, por parte de las especies que habitan nidos y madrigueras. En algunos casos de foresia sobre invertebrados, el insecto transportador es, asimismo, utilizado como presa o lo son los parásitos que éste pueda transportar consigo (ello sucede principalmente con especies pertenecientes a las familias menos evolucionadas); ante esto, se han planteado dudas sobre la finalidad con que el pseudoscorpión utiliza la foresia: ¿transporte o predación?. Posiblemente se trate de un caso u otro dependiendo de la identidad de ambas especies, transportadora y transportada.

Como enemigos de los pseudoscorpiones se han citado algunos casos de parasitismo. VACHON (1949) y HARVEY (1981) describen dos casos de pseudoscorpiones parasitados por nematodos. Mejor conocido es el parasitismo en algunos Neobisium por el icneumónido Obisiphaga (Hemiteles) sternoptera (Ichneumonidae, Cryptinae), especializada en la deposición larvaria en el interior de los nidos de los diferentes estadios ninfales del pseudoscorpión (WEYGOLDT, 1969; HEURTAULT, 1973).

En lo que respecta a los predadores de los pseudoscorpiones, los estudios son mucho más escasos. Para WEYGOLDT (1969), los pseudoscorpiones no tienen enemigos especiales, pero cualquier animal que busca en la hojarasca o bajo las cortezas de árboles a pequeñas presas, como es el caso de arañas, quilópodos, ácaros, abejas, hormigas o aves, puede incluir en dieta pseudoscorpiones, accidentalmente. HEURTAULT (1973) cita al escorpión Euscorpius carpathicus como probable enemigo del pseudoscorpión Neobisium caporiaccoi. Faltan, desde luego, estudios sobre la incidencia de estas capturas en el control de la población de pseudoscorpiones y en qué porcentaje de la dieta pasan éstos a formar parte.

Atendiendo al consumo de pseudoscorpiones por parte de vertebrados, que constituye una de las partes de este trabajo, son escasos los datos acumulados. Las obras generales de los más prestigiosos pseudoscorpionólogos, como Beier, Chamberlin, Vachon, salvo la ya citada de Weygoldt, no mencionan nada sobre el particular. SCHAWALLER (1981), en su relación bibliográfica sobre pseudoscorpiones desde 1890 a 1979, recoge dos artículos de CHAMBERLIN $(1925,1934)$, en los que se analiza el material procedente de los contenidos estomacales de sapos de la especie Bufo y del caprimulgiforme Chordeiles minor, pero contemplados desde el punto de vista exclusivamente sistemático; en dicho trabajo se mencionan, asimismo, otros dos artículos de MILSTEAD (1958) y de NICULESU (1963) sobre la alimentación de saurios y anuros, a partir de los restos encontrados en sus estómagos y donde se registran datos porcentuales de la presencia de pseudoscorpiones. 
Se ha hecho evidente para los autores de este artículo, que existen muchos más datos que los anteriormente citados sobre la incorporación de pseudoscorpiones a la dieta de los vertebrados, principalmente de aves, batracios y reptiles. La búsqueda de artículos en boletines ornitológicos y herpetológicos, nos ha resultado una buena fuente de información. Así, se han podido reunir una serie de ellos de interés sobre el tema y a los que, a continuación, hacemos referencia; no se trata aquí, sin embargo, de confeccionar una lista exhaustiva con todos los trabajos que tratan sobre el particular, que suponemos muy numerosos y dispersos, sino de correlacionar los datos extraídos de aquéllos con los que aquí se ofrecen. Creemos que es una aportación interesante a un aspecto poco conocido de la ecología de los pseudoscorpiones.

Los pseudoscorpiones han sido citados como componentes de la dieta de las aves, batracios y reptiles y cuya presencia ha sido reconocida mediante examen de los contenidos estomacales, por diversos métodos sin que, aparentemente, ninguno de ellos modifique sustancialmente los resultados.

En general podemos afirmar que los pseudoscorpiones componen una parte mínima de la dieta de los vertebrados, entre un $0.2 \%$ y un $6 \%$ del total de presas capturadas, aunque en algunos casos aislados la proporción puede incrementarse notablemente (s/ $20 \%$ en un tubo con contenidos estomacales de Acanthodactylus erythrurus). Ello parece debido tanto a la proporción con que los pseudoscorpiones aparecen en los diferentes nichos con respecto al total de la población de artrópodos, como a su comportamiento ecléctico con un breve desarrollo de su actividad al aire libre ya que, normalmente, se hallan refugiados entre la hojarasca o el musgo, en el interior de las grietas o bajo las piedras o las cortezas de los árboles.

Entre las aves, las que observan mayor incidencia predadora sobre los pseudoscorpiones son los pequeños pájaros insectívoros y, entre éstos, tanto aquellos que buscan su alimento en el suelo, tales como el gorrión común (Passer domesticus) y el miná pastoril (Acridotheres tristis) (RALPH, 1985) o los que, como la curruca cabecinegra (Sylvia melanocephala), son predominantemente arborícolas (TEJERO et al., 1983). La presencia de una buena colección de pseudoscorpiones en el tracto digestivo de un caprimulgiforme (Chordeiles minor) (CHAMBERLIN, 1934), aves que se alimentan en vuelo de insectos alados y nunca de artrópodos del suelo, nos causaba una cierta perplejidad, mas el mismo Chamberlin ha dado, indirectamente, la respuesta: uno de los paratipos de las especies descritas había sido previamente recogido forético sobre un cerambícido; de este modo original, transportados por un insecto, se incorporaban a la dieta del ave.

Los batracios son otro grupo en los que se ha constatado la presencia de pseudoscorpiones en su alimentación. De entre los anuros, el género Bufo (CHAMBERLIN, 1925, GUYETANT, 1967, GITTINS, 1987) y el género $A l y$ tes (MELLADO, 1976) los incorporan debido al desarrollo de una actividad más terrestre que otras especies de hábitos más acuáticos (Rana ridibunda, NICULESU, 1963).

Otro tanto sucede con los urodelos: los pseudoscorpiones hacen su aparición como componentes de la alimentación de las especies que se desenvuelven predominantemente sobre la hojarasca del suelo forestal, como algunas salamandras (Eurycea quadridigitata, McMILLAN y SEMLITSCH, 1980; Plethodon jordani, MITCHELL, 1986). 
En el grupo de los reptiles son, naturalmente, los pequeños saurios los que, con mayo frecuencia, capturan pseudoscorpiones para incorporarlos en mayor o menor medida a su régimen alimentario (Anolis opalinus, FLOYD y JENSSEN, 1983; Acanthodactylus erythrurus, Psammodromus algirus, VALVERDE, 1967; mismas especies, SEVA, 1982).

Los pseudoscorpiones son, verdaderamente, un tipo de arácnidos bien escaso y los porcentajes de presencia, tanto en hojarasca, como partes aéreas de arbustos -entre un $0,1 \%$ y un $2 \%$ del número total de microartrópodos (ATHIAS, 1971; COLOMER, 1981)-, resultan similares a los de su presencia en estómagos de algunas especies, e inferiores a los que cabría esperar en una dieta no direccional. A pesar de su tamaño, que en la gran mayoría de los predadores de cuya dieta forman parte, ocupa un lugar muy por debajo de la media dimensional y su presencia por encima de los porcentajes previstos es indicativa de una selección por parte del depredador. Esa selección responde, por una parte a su movilidad en la actividad diurna, y por otra, a la dimensión espacial de su nicho ecológico.

La relación espacial que establece la lagartija colirroja ( $A$. erythrurus) en torno a los grandes arbustos de Thymelaea hirsuta explica la ocurrencia del pseudoscorpión Olpium pallipes, tanto en los mangueos efectuados en ramas bajas de este arbusto, como en bastantes contenidos estomacales de $A$. erythrurus. En efecto, $O$. pallipes desarrolla su actividad sobre ramas y hojas de Thymelaea hirsuta en un estrato altitudinal que no sobrepasa los $15-20 \mathrm{cms}$., a los que tiene acceso $A$. erythrurus, sobre el que se ha demostrado un comportamiento no muy trepador.

La escasa presencia del pseudoscorpión en estómagos y heces del saurio en los meses primaverales, remarca el calificativo de presa «accidental», como puedan ser ejemplares de Ammobius rufus, de similar tamaño, a pesar de que la frecuencia en las muestras de mangueos es superior a la de otros meses. Por contra, la presencia a partir del mes de junio en los estómagos es masiva, ya que pasa a ser presa «probable», por la gran ausencia de grandes grupos. Recuérdese (SEVA, 1982) que $A$. erythrurus observa un poderoso declive en el volumen alimentario a partir de junio y que, incluso, accede a restos de detritos procedentes de la actividad humana. En esta etapa de su alimentación los insectos de clara manifestación gregaria y escasa movilidad constituyen el mayor porcentaje de su dieta (formícidos, hasta un $75 \%$ sobre el número total de presas en algunos estómagos).

En el caso de Psammodromus algirus, saurio que presenta un rango de ocupación vertical del espacio mucho mayor (SEVA, 1984) y del que se puede extraer el calificativo de «trepador» sobre arbustos de Crucianella maritima y Thymelaea hirsuta, tanto $O$. pallipes como Hysterocherifer sp., constituyen presas muy "accidentales», más debido a sus cortos traslados que a su representatividad dentro del espectro alimentario en los estratos altos arbustivos, de los que este saurio obtiene un gran número de ortópteros en los meses estivales.

En general, sobre la incidencia de la predación por vertebrados en las poblaciones de pseudoscorpiones, podemos aventurar las siguientes conclusiones:

- Los pseudoscorpiones son consumidos por los vertebrados en pequeñas cantidades, algunas simplemente testimoniales, otras no poco importantes 
y aproximadamente similares a la proporción en que éstos se encuentran respecto a la población total de invertebrados.

- La falta de familiarización con determinadas estructuras de los pseudoscorpiones hace que las personas encargadas de la determinación de los contenidos de estómagos y/o heces puedan confundirlas como pertenecientes a otros artrópodos o no lleguen a identificarlas, desvirtuando de este modo los datos reales sobre su presencia y frecuencia. Una sola pata ambulatoria de pseudoscorpión provista del exclusivo arolio es tan buen indicador como una pinza del pedipalpo, que es la normalmente utilizada por los profanos.

- Parecen confirmarse algunos comportamientos individuales, y sobre todo, estacionales en los depredadores. Ello explicaría la elevada proporción de pseudoscorpiones en algunos estómagos de $A$. erythrurus y hasta la presencia de un solo ejemplar de dos especies diferentes de quernetos, posiblemente con hábitos distintos.

- Resulta de alto interés el estudio de los pseudoscorpiones aparecidos en los restos alimentarios de vertebrados. No únicamente para el estudio ecológico del comportamiento de ambos grupos, lo que ya justificaría la atención, sino también desde el punto de vista sistemático, pues se accede de este modo a especies que, por sus hábitos ubiquistas o foréticos, no son frecuentemente capturadas por los pseudoscorpionólogos; basta recordar, a tal efecto, que por este sistema CHAMBERLIN $(1925,1934)$ describió varios géneros y especies inéditos. 


\section{BIBLIOGRAFÍA}

ATHIAS, F. (1971). Etude quantitative preliminaire des microarthropodes du sol. Recherches ecologiques dans la savane de Lamto (Côte d'Ivoire). Bull. Ecol. 1 ere serie: 73-87.

CALLAINI, G. (1986). Su alcune specie di Cheliferidae della regione mediterranea. Boll. Mus. Civ. St. Nat. Verona, 13: 273-294.

CHAMBERLIN, J. C. (1925). On a collection of pseudoscorpions from the stomach contents of toads. Univ. Calif. Publ. Ent. 3: 327-332.

- (1934). On two species of false scorpions collected by birds in Montana, with notes on the genus Dinocheirus (Arachnida-Chelonethida). Pan-Pacific Entomol., 10: 125-132.

COLOMER, S. y E. ALBERTUS (1981). Distribución y abundancia relativa de los microartrópodos edáficos en la Dehesa de la Albufera de Valencia. Bol. Est. Cent. Ecología X, 19: 89-97.

FLOYD, H. B. y T. A. JENSSEN (1983). Food habits of the Jamaican lizard Anolis opalinus: resource partitioning and seasonal effects examined. Copeia, 2: 319-331.

GILBERT, O. (1951). Observations on the feeding of some British false scorpions. Proc. Zool. Soc. London, 121: 547-555.

GITTINS, S. P. (1987). The diet of the common toad (Bufo bufo) around a pond in Mid-Wales. Amphibia-Reptilia, 8 (1): 13-17.

GUYETANT, R. (1967). Étude de l'alimentation de jeunes batraciens anoures durant la saison estivale. Ann. Sci. Univ. Besançon, 3: 69-78.

HARVEY, M. S. (1981). A parasitic nematode (Mermithidae) from the pseudoscorpion «Sternophorus» hirsti Chamberlin (Sternophoridae). Journ. Arachnology, 10: 192.

HEURTAUL.T, J. (1973). Contribution a la connaisance biologique et anatomo-physiologique des Pseudoscorpions. Bull. Mus. Hist. Nat., (3) 124: 561-670.

- (1976). Nouveaux caractères taxonomiques pour la sous-famille des Olpiinae (Arachnides, Pseudoscorpions). Note preliminaire. C. R. Coll. Arachn. France, Les Eyzies: 62-73.

- (1979). Complément à la description de Olpium pallipes Lucas, 1845, type de la famille Olpiidae (Arachnides, Pseudoscorpions). Revue suisse Zool., 86 (4): 925-931.

- (1980). Données nouveiles sur les genres Xenolpium, Antiolpium, Indolpium et Euryolpium (Arachnides, Pseudoscorpions). Rev. suisse Zool., 87 (1): 143-154.

McMILLAN, M. A. y R. D. SEMLITSCH (1980). Prey of the dwarf salamander, Eurycea quadridigitata in South Carolina. Journal of Herpetology, 14 (4): 414-426.

MAHNERT, V. (1982). Die Pseudoskorpione (Arachnida) Kenyas. 3. Olpiidae. Monit. Zool. Ital. 11: $263-304$.

MELLADO, J. (1976). Sobre la alimentación del sapo partero ibérico (Alytes cisternasii Boscá) (Anura: Discoglosiidae). Bol. Est. Cent. Ecologia, 5 (9): 81-84.

MILSTEAD, W. M. (1958). A list of the arthropods found in the stomach of whiptail lizards from four stations in southwestern Texas. Texas $J$. Sci., 10: 443-446.

MITCHELL, J. C. y J. A. TAYLOR (1986). Predator-prey relationships in a North Carolina population of Plethodon jordani. Journal of Herpetology, 20 (4): 562-566.

NICULESU, F. y I. E. FUHN (1963). Étude de la nourriture chez la grenouille verte (Rana r. ridibunda) dans la réservation naturelle «I. Mai». Studii Cercet. Stiint. Iaçi, (Biol. stiint. agric.), 14: 193-211.

RALPH, C. P.; NAGATA, S. E. y C. J. RALPH (1985). Analysis of droppings to describe diets of small birds. J. Field Ornithol., 56 (2): 165-174.

SCHAWALLER, W. (1980). Bibliographie der rezenten und fossilen Pseudoscorpionidea 18901979 (Arachnida). Stuttgarter Beitr. Naturk. Ser. A., 338: 1-61.

SEVA, E. (1982). Taxocenosis de Lacértidos en un arenal costero alicantino. Pub. Universidad de Alicante. $317 \mathrm{p}$.

- (1984). Reparto de recursos en dos especies psammófilas de saurios: Acanthodactylus erythrurus y Psammodromus algirus. Arenal costero de Alicante. Mediterránea, 7: 5-25.

TEJERO, E., CAMACHO, I. y M. SOLER (1983). La alimentación de la curruca cabecinegra (Sylvia melanocephala, Gmelin 1788) en olivares de la provincia de Jaén (otoño-invierno). Doñana, Acta vertebrata, 10 (1): 133-153.

VACHON, M. (1949). Ordre des Pseudoscorpions. In: Grassé, P. Traité de Zoologie, 6: 437-481. París.

- (1966). Olpium minnizoides nouvelle espèce de pseudoscorpion Olpiidae habitant l'île Hasikaya (Sud d'Arabie). Annals Mag. Nat. Hist. (13) 9: 183-188.

VALVERDE, J. A. (1967). Estructura de una comunidad de vertebrados terrestres. Monografías de la Estación Biológica de Doñana. 1: 1-219.

WEYGOLDT, P. (1969). The biology of pseudoscorpions. Harvard books in Biology, 6: 1-145. 